\title{
Comprensión de futuros profesores de ciencia sobre aspectos epistémicos de la naturaleza de la ciencia en cuatro controversias de historia de la ciencia
}

\author{
Pre-service science teachers' understanding of epistemic aspects of the nature of \\ science in four controversies from the history of science
}

\section{Compreensão de futuros professores de ciência sobre aspectos epistêmicos da natureza da ciência em quatro controvérsias da história da ciência}

\author{
José Antonio Acevedo-Díaz ${ }^{1}$ \\ María del Mar Aragón-Méndez² \\ Antonio García-Carmona ${ }^{3}$
}

Recibido: mayo de 2018

Aceptado: agosto de 2018

Para citar este artículo: Acevedo-Díaz, J. A., Aragón-Méndez, M. M. y García-Carmona, A. (2018). Comprensión de futuros profesores de ciencia sobre aspectos epistémicos de la naturaleza de la ciencia en cuatro controversias de historia de la ciencia. Revista Científica, 33(3), 344-355. Doi: https://doi.org/10.14483/23448350.13355

\section{Resumen}

Se presenta un análisis global de los resultados de cuatro estudios que evalúan la comprensión de futuros profesores de ciencia de Educación Secundaria sobre aspectos epistémicos de la naturaleza de la ciencia (NDC). Para ello, se utilizan cuatro controversias de historia de la ciencia (HDC) como contexto de enseñanza/aprendizaje, cuya implementación en el aula se lleva a cabo mediante un enfoque crítico y reflexivo. El análisis realizado se concreta en determinar las referencias en las respuestas de los futuros profesores de ciencia a los distintos indicadores epistémicos de las rúbricas de evaluación, así como en la cantidad de veces que esos indicadores son aludidos en total. Los resultados obtenidos permiten concluir que los futuros profesores de ciencia, en general, mejoran significativamente su comprensión sobre la influencia de los aspectos epistémicos de la NDC en las cuatro controversias de HDC empleadas.

Palabras clave: aspectos epistémicos, formación inicial del profesorado de ciencia, historia de la ciencia, naturaleza de la ciencia, procesos de la ciencia, productos de la ciencia.

\begin{abstract}
This article presents a global analysis of the results from four studies on pre-service secondary science teachers' understanding of epistemic aspects of the nature of science (NOS). To this end, four controversies from the history of science (HOS) were used as teaching/learning context, and whose implementation in class was conducted through a critical and reflective approach. The analysis focused on
\end{abstract}


determining the references that pre-service science teachers made in their responses to the different epistemic indicators established in the assessment rubrics, and the number of times that these were alluded. The findings obtained allow to conclude that, in general, the pre-service science teachers significantly improved their understanding on the influence of the epistemic aspects of NOS in the four controversies of HOS which were employed.

Keywords: epistemic aspects, history of science, nature of science, pre-service science teacher education, science processes, science products.

\section{Resumo}

Este artigo apresenta uma analise global sobre os resultados de quatro estudos que avaliaram a compreensão de futuros professores de ciência em formação inicial, sobre aspectos epistémicos da natureza da ciência (NDC). Para isso, quatro controvérsias da história da ciência (HDC) foram utilizadas como contexto de ensino/aprendizagem, cuja implementação em sala de aula foi realizada por meio de uma abordagem crítica e reflexiva. A análise buscou determinar as referências feitas pelos professores de ciência em formação inicial em suas respostas, aos diferentes indicadores epistêmicos estabelecidos nas rubricas de avaliação, assim como o número de vezes em que esses foram mencionados. Os resultados obtidos permitem concluir que, em geral, os professores de ciências em formação inicial melhoraram significativamente sua compreensão sobre a influência dos aspectos epistêmicos da NDC nas quatro controvérsias das HDC que foram utilizadas.

Palavras-chaves: aspectos epistêmicos, formação inicial de professores da ciência, história da ciência, natureza da ciência, processos da ciência, produtos da ciência.

\section{Introducción}

Existe cierto consenso mundial en considerar la naturaleza de la ciencia (NDC) como un conocimiento clave para la alfabetización científica de la ciudadanía (Acevedo-Díaz y García-Carmona 2016a; Hodson, 2014; Next Generation Science Standards $\left[\mathrm{NGSS}^{4}, 2013\right.$; Organisation for Economic Co-operation and Development $[\mathrm{OECD}]^{5}$, 2017; Vázquez-Alonso y Manassero-Mas, 2018; entre otros). Una buena comprensión de la NDC tiene valor per se en la cultura científica deseable para la ciudadanía (Acevedo y García-Carmona, 2017); además de un papel esencial para enriquecer la educación científica (Acevedo, 2008).

Las razones de esa consideración son diversas, pero una es que las personas recurren esencialmente a sus conocimientos, más o menos informados, sobre la NDC (Shamos, 1995) cuando valoran asuntos públicos relacionados con la ciencia y la tecnología. Por tanto, una buena comprensión de la NDC ayudaría a que esas valoraciones estén mejor fundamentadas (Acevedo-Díaz y García-Carmona, 2017). En esta línea, el programa marco Horizonte 2020 para la Investigación e Innovación en la Unión Europea $^{6}$ establece entre sus objetivos básicos promover "La participación ciudadana en la ciencia, de manera que los ciudadanos desarrollen intereses y capacidades hacia la ciencia, que les permitan participar activamente en actividades científicas" (s.f.). Esta iniciativa ha trascendido recientemente al ámbito de la educación científica formal con el desarrollo de proyectos como IRRESISTIBLE (Including Responsible Research and innovation in cutting Edge Science and Inquiry-based Science education to improve Teacher's Ability of Bridging Learning Environments), financiado por la Unión Europea

4. Los NGSS abordan la NDC en el apéndice H, titulado Understanding the Scientific Enterprise: The Nature of Science in the Next Generation Science Standards. La matriz de la NDC incluye ocho temas.

5. El documento de la OCDE lo hace de una manera simple, con muy pocas preguntas propias de la NDC, y de modo incompleto porque enfoca la NDC exclusivamente a asuntos epistémicos.

6. Con el eslogan "ciencia con y para la sociedad", este proyecto europeo propone que la alfabetización científica debe encaminarse a que la ciudadanía se interese por la ciencia y sea partícipe de manera activa, crítica y responsable con los distintos actores e instituciones que propician el desarrollo científico-tecnológico (véase Horizonte 2020 para la Investigación e Innovación en la Unión Europea, s.f.). 
dentro del programa Science in Society y en el que participan 10 países europeos (Laherto et al., 2018).

Hasta ahora la propuesta de enseñanza sobre NDC que ha dominado en la bibliografía internacional es la que viene dada por los siete principios de Lederman (2007). Estos constituyen una lista restringida a aspectos epistémicos de la naturaleza del conocimiento científico; esto es, aspectos racionales o cognitivos relativos a la naturaleza epistémica de los productos elaborados por la ciencia. Sin embargo, durante la presente década está teniendo lugar una discusión relevante sobre qué aspectos de NDC serían los más adecuados para el currículo de ciencia en la educación preuniversitaria (Acevedo y García Carmona, 2016a; Acevedo-Díaz, García-Carmona y Aragón-Méndez, 2017a; Allchin, 2011; Dagher y Erduran, 2016; Hodson y Wong, 2017; Martins, 2015; entre otros).

\section{Marco teórico}

La posición que adoptamos sobre qué enseñar de NDC se basa en una perspectiva holística más acorde con la complejidad de la construcción del conocimiento científico (Acevedo-Díaz, García-Carmona y Aragón-Méndez, 2017a), coherente con la práctica científica real (Acevedo y García Carmona, 2016a), y que tenga en consideración el continuo existente entre los contextos de descubrimiento y de justificación ${ }^{7}$ (Acevedo-Díaz, 2017). Esta perspectiva incluye aspectos epistémicos de la NDC, relativos al conocimiento científico producido y a los procesos de indagación científica (Driver, Leach, Millar y Scott, 1996) ${ }^{8}$, así como otros aspectos no-epistémicos internos y externos a la comunidad científica. En este artículo no se tratarán los aspectos no-epistémicos de la NDC, los cuales suelen ser los más comunes entre las diferentes ciencias (Acevedo-Díaz, 2018).

El enfoque que se ha seguido en la enseñanza de la NDC ha sido explícito, reflexivo y contextualizado. Explícito, en el sentido de que la NDC se incluye de forma planificada y evaluable como un contenido curricular específico (Acevedo-Díaz, 2009). Reflexivo, en tanto que se plantea a los estudiantes mediante cuestiones para su análisis críti$\mathrm{CO}^{9}$ (Clough, 2011). Y contextualizado, en este caso mediante la HDC, utilizando narraciones de controversias científicas elaboradas por los propios autores de este artículo (Acevedo y García-Carmona, 2016b, 2016c y 2017; Acevedo-Díaz, García-Carmona y Aragón-Méndez, 2016a, 2016b y 2017b).

El propósito de este artículo es mostrar la comprensión global lograda por futuros profesores de ciencia de Educación Secundaria sobre diversos aspectos epistémicos de NDC, a partir del análisis crítico-reflexivo y los argumentos correspondientes, en el aula de cuatro casos de historia de la ciencia (HDC). Los casos históricos que fueron analizados y discutidos por los futuros docentes fueron los siguientes: "Semmelweis y la fiebre puerperal"; "La controversia entre Pasteur y Liebig sobre la fermentación"; "la controversia entre Pasteur y Pouchet sobre la generación espontánea" y "Rosalind Franklin y la elucidación de la estructura del ADN".

\section{Metodología}

\section{Contexto académico}

En España es necesario obtener un título de postgrado denominado Máster Universitario en Profesorado de Educación Secundaria (en adelante, MAES) para poder ejercer la profesión docente en Educación Secundaria. Este consta de: 1) un módulo

Terminología introducida, circa 1930, por el filósofo positivista Reichenbach para diferenciar la génesis de los descubrimientos científicos (ciencia privada) de los resultados finales de la investigación científica (ciencia publicada).

8. Es muy importante no confundir el conocimiento de procedimientos científicos, propios de la indagación científica, con el conocimiento epistémico sobre los procedimientos científicos empleados en la indagación científica; el segundo es de naturaleza epistémica, mientras que el primero no (Acevedo-Díaz, García-Carmona y Aragón-Méndez, 2017a).

9. Un enfoque acorde con el pensamiento crítico, que tal y como lo definen Norris y Ennis (1989) es: "[...] una actividad práctica y reflexiva, cuya meta es una creencia o una acción sensata; [...] una forma de pensamiento racional, reflexivo, enfocado a decidir en qué creer o qué hacer.". 
genérico para todas las especialidades docentes, que proporciona una formación psicopedagógica general; 2) un módulo orientado a la formación específica de cada especialidad docente; y 3) un módulo de prácticas en centros educativos. Para acceder al MAES, los estudiantes deben poseer, al menos, un título de graduado universitario.

El currículo de ciencia para Educación Secundaria en España es muy poco explícito en sugerir la NDC como un contenido esencial de la educación científica. Sin embargo, el plan de estudios del MAES establece que los estudiantes de profesorado deben adquirir una comprensión básica sobre NDC y su enseñanza.

\section{Participantes}

La implementación de la controversia relativa a Semmelweis y la fiebre puerperal se realizó, durante el curso 2015-16, en la Universidad de Cádiz con estudiantes de la especialidad docente de Biología y Geología del MAES. Participaron 12 mujeres y 5 hombres, graduados en Biología, Ciencias Ambientales, Ciencias del Mar, Farmacia y Bioquímica. La asignatura para desarrollar la actividad fue Aprendizaje y Enseñanza de la Biología y Geología, de 12 créditos, impartida por la coautora de este artículo; más concretamente, en la unidad Análisis curricular.

La implementación de la controversia entre Pasteur y Liebig sobre la fermentación se llevó a cabo, durante el curso 2015-16, en la Universidad de Sevilla con estudiantes de la especialidad docente de Física y Química del MAES. Los participantes fueron 10 mujeres y 9 hombres, entre los que había 2 graduados en Ciencias Físicas, 2 en Biotecnología, 13 en Ciencias Químicas y 2 en Ingeniería Química. La asignatura para desarrollar la actividad fue Aprendizaje y Enseñanza de las materias de Física y Química, que cuenta con 60 horas presenciales, impartida por el segundo autor de este artículo; concretamente, dentro de una unidad breve denominada ¿Qué es la Ciencia? Algunas reflexiones sobre la naturaleza de la ciencia y sus implicaciones didácticas.
Las implementaciones de las controversias entre Pasteur y Pouchet sobre la generación espontánea y de la participación de Rosalind Franklin en la elucidación de la estructura del ADN se hicieron, durante el curso 2016-17, en la Universidad de Cádiz con estudiantes de la especialidad docente de Biología y Geología del MAES. Los participantes en ambas fueron los mismos, 14 mujeres y 3 hombres, licenciados o graduados en Ciencias Ambientales, Ciencias del Mar, Biología, Biotecnología, Biomedicina, Bioquímica y Veterinaria. Algunos de ellos tenían doble titulación, como Ciencias del Mar y Ciencias Ambientales. La asignatura para desarrollar las dos actividades fue Complementos de Formación disciplinar de Biología y Geología, impartida por la coautora de este artículo; concretamente, en el bloque Naturaleza e Historia de la Ciencia. Los contenidos de este bloque giran en torno a diferentes episodios de HDC, sobre todo de Biología y Geología, como hilo conductor para la formación de los futuros profesores en la NDC en aspectos epistemológicos, ontológicos y sociológicos de la ciencia.

\section{Breve descripción de la propuesta de enseñanza}

Los aspectos epistémicos de la NDC abordados entre las cuatro controversias se refieren tanto a los productos de la ciencia (tabla 1) como a los procesos que tienen lugar en la construcción del conocimiento científico (tabla 2). A continuación, se señalan estos aspectos, indicándose asimismo las controversias en las que se tratan.

En todos los casos, los estudiantes se organizaron en grupos pequeños de trabajo para el desarollo de las actividades. Se emplearon unas cinco horas en cada implementación, que se estructuraron en tres fases: 1 ) lectura del relato de la controversia de HDC y respuestas a diversas cuestiones de NDC, a partir de la reflexión y discusión entre los miembros de cada grupo, que se redactaron en un informe inicial; 2) sesión con el grupo-clase, dirigida por los educadores correspondientes para que los grupos compartieran y debatieran sus 
respuestas iniciales; y 3) conclusiones finales de los grupos tras la fase anterior, que se concretaron en la revisión y reelaboración/ampliación de sus respuestas iniciales. Estas respuestas también se registraron por los grupos en un informe final que entregaron, junto con el informe inicial, para su evaluación.

\section{Instrumentos de análisis de datos y evaluación}

Respecto a la evaluación de aprendizajes sobre NDC, nos alineamos con Allchin (2011), quien propone un enfoque contextual mediante el análisis interpretativo de casos actuales e históricos de la ciencia. Por tanto, la metodología de evaluación de los aspectos de NDC abordados con la actividad tiene un carácter cualitativo, está contextualizada mediante controversias de HDC, es de índole interpretativa y se basa en el uso de rúbricas para el análisis de datos.

Para la evaluación de las respuestas a cada una de las cuestiones planteadas se estableció una escala de progresión que permitiera el análisis del nivel de aprendizaje alcanzado por los estudiantes. Se decidieron cinco niveles, de 0 a 4, correspondiendo el máximo aprendizaje conseguido al nivel

Tabla 1. Naturaleza epistémica de los productos de la ciencia.

\begin{tabular}{|c|c|}
\hline Productos de la ciencia & Controversias en las que se tratan \\
\hline Características de las teorías científicas. & Fiebre puerperal; Fermentación. \\
\hline Diferencias entre leyes y teorías científicas. & Fermentación. \\
\hline $\begin{array}{l}\text { Diferencias en la interpretación científica de un mismo } \\
\text { fenómeno. }\end{array}$ & Fermentación; Generación espontánea. \\
\hline Provisionalidad de las teorías científicas. & Fermentación; Generación espontánea. \\
\hline Dominancia de algunas teorías científicas sobre otras. & Fermentación. \\
\hline $\begin{array}{l}\text { Carácter tentativo y dinámico del conocimiento } \\
\text { científico. }\end{array}$ & Fermentación; Generación espontánea. \\
\hline
\end{tabular}

Fuente: elaboración propia de los autores.

Tabla 2. Naturaleza epistémica de los procesos de la ciencia.

\begin{tabular}{|c|c|}
\hline Procesos de la ciencia & Controversias en las que se tratan \\
\hline Observación vs. inferencia & Fiebre puerperal. \\
\hline Metodologías científicas. & Fiebre puerperal; Fermentación; ADN. \\
\hline Papel de las hipótesis. & Fiebre puerperal. \\
\hline Creatividad e imaginación. & Fiebre puerperal; Fermentación; ADN. \\
\hline Papel de la experimentación en la ciencia. & ADN; Generación espontánea. \\
\hline Papel de los errores en el desarrollo de la ciencia. & Fermentación; Generación espontánea. \\
\hline $\begin{array}{l}\text { Influencia de las creencias personales, actitudes y } \\
\text { habilidades de los científicos. }\end{array}$ & Fermentación; Generación espontánea. \\
\hline Papel de los esquemas de clasificación. & Fermentación. \\
\hline $\begin{array}{l}\text { Interés de las controversias científicas para el avance de la } \\
\text { ciencia. }\end{array}$ & Fermentación. \\
\hline Diseños de investigación y resultados experimentales. & ADN; Generación espontánea. \\
\hline $\begin{array}{l}\text { Influencia de la especialidad del científico en la } \\
\text { planificación y desarrollo de una investigación científica. }\end{array}$ & ADN. \\
\hline Pregunta que dirige la investigación y objetivos perseguidos. & ADN. \\
\hline Modelos y modelización en la ciencia. & ADN. \\
\hline
\end{tabular}

Fuente: elaboración propia de los autores. 
4. Los indicadores que definen el nivel máximo se establecieron tomando como punto de partida el texto de la narración de HDC, los puntos de vista actuales sobre la NDC y la información proporcionada por las respuestas de los grupos. Estos indicadores se modificaron y reestructuraron varias veces hasta que los autores de este trabajo Ilegaron a un consenso. Los diferentes niveles de progresión y los indicadores correspondientes se recogieron luego en una rúbrica de evaluación para cada controversia empleada. Como ejemplo, se muestra la parte de la rúbrica correspondiente a las cuestiones relativas a la controversia sobre el caso de Rosalind Franklin y la estructura del ADN (tabla 3).

Tabla 3. Rúbrica de evaluación de aspectos epistémicos de la NDC en el contexto del caso Rosalind Franklin y la doble hélice del ADN.

\begin{tabular}{ll}
\hline Contenido de NDC & Nivel 4 (máximo) \\
\hline C1. Pluralidad de & Se explica que no hay un método científico úni- \\
metodologías de & co y se describen las dos metodologías identifi- \\
las investigaciones & cables en la narración histórica: \\
científicas. & 1) La empírica sistemática de Franklin. \\
& 2) La de Watson y Crick basada en la \\
& elaboración de un modelo funcional. \\
& 3) Se argumenta, razonadamente, que ninguna \\
& metodología es per se mejor que la otra.
\end{tabular}

C2. Objetivos de las investigaciones científicas.
Se explica que los objetivos eran diferentes y en la explicación se señalan tres de las razones siguientes:

1) Se explica el objetivo de Franklin.

2) Se explica el objetivo de Watson y Crick.

3) Se relacionan los objetivos con la formación de los científicos.

4) Se relacionan los objetivos con las metodologías empleadas.

5) Se relacionan los objetivos con las prioridades de los centros de investigación.

\section{Niveles 3-0}

Nivel 3: se explica que no hay un método científico único, pero solo se describe una de las dos metodologías [1) o 2)]Se argumenta, razonadamente, que ninguna metodología es per se mejor que la otra [3)].

Nivel 2: se explica que no hay un método científico único, pero no se describe ninguna de las metodologías [ni 1) ni 2)].

Se argumenta, razonadamente, que ninguna metodología es per se mejor que la otra [3)]. Nivel 1: Se considera que son posibles varias metodologías científicas y, aunque se describan, se asume que una de ellas es per se mejor que otra.

Nivel 0: se considera que solo hay un único método científico válido.

Nivel 3: se explica que los objetivos eran diferentes y en la explicación se señalan dos de las razones anteriores.

Nivel 2: se explica que los objetivos eran diferentes, y en la explicación se señala una de las razones anteriores.

Nivel 1: se explica que los objetivos eran diferentes, pero no se aportan argumentos válidos. Nivel 0: no se identifican diferencias significativas entre los objetivos.

Nivel 3: se indican dos fortalezas de forma justificada.

Nivel 2: se indica una fortaleza y se justifica.

Nivel 1: se indica alguna fortaleza, pero no se justifica o la justificación no es válida.

Nivel 0: no se indica ninguna fortaleza.
C3. Fortalezas del modelo de Watson y Crick.
Se indican, de forma justificada, al menos tres fortalezas; por ejemplo:

1) Explica la estructura del ADN.

2) Permite dar explicaciones y hacer predicciones sobre el código genético.

3) Permite establecer una hipótesis fecunda para investigaciones futuras.

4) Da respuesta a problemas multidisciplinares.

Fuente: elaboración propia de los autores. 
La validez y fiabilidad de este sistema de evaluación fueron establecidas mediante procesos iterativos de análisis inter e intra-jueces, realizados por los autores, y el uso de indicadores de baja inferencia (en este caso, citaciones textuales de las respuestas de los grupos a las preguntas planteadas); ambos muy recurrentes y apropiados en investigaciones cualitativas como esta (Seale, 1999).

Para abordar el objetivo de este trabajo, se ha realizado un análisis del número total de referencias de los grupos a los indicadores epistémicos, establecidos en las rúbricas de evaluación, y de la cantidad de veces que tales indicadores fueron aludidos como argumentos en conjunto.

\section{Resultados}

El número total de indicadores epistémicos citados de las rúbricas (tabla 4) fue más elevado en los informes finales (86\%) que en los iniciales (68\%). Asimismo, la cantidad de veces que se citaron tales indicadores en total aumentó de forma notable, aproximadamente el doble, en los informes finales respecto de los iniciales. Puede comprobarse que este incremento no es muy diferente en cada una de las cuatro controversias de HDC implementadas. De igual manera, cabe señalar que, si bien esta comprensión ya se manifestó de manera suficiente en los informes iniciales de los grupos, mejoró en los informes finales. En consecuencia, estos resultados muestran que los futuros profesores de
Ciencia en educación secundaria son capaces de apreciar, comprender y argumentar la influencia de los aspectos epistémicos de la NDC en las controversias tratadas.

En la tabla 5 se muestran algunos ejemplos de la variedad de argumentos usados en las respuestas de los informes finales de los grupos a tres cuestiones epistémicas de la controversia entre Pasteur y Pouchet sobre la generación espontánea. A saber: 1) el papel de las creencias teóricas en la interpretación de las observaciones; 2) el papel de los diseños experimentales en los resultados obtenidos; y 3) la presencia o ausencia de experimentos cruciales durante la controversia.

En la obtención de los resultados finales hay que resaltar la influencia que tuvo la sesión con el grupo-clase en la que se debatieron las ideas que los grupos plasmaron en los informes iniciales. Para ello, el papel de los educadores respectivos durante esta sesión resultó clave en cada implementación.

Durante la puesta en común del grupo-aula en cada implementación el educador intervino como moderador, formulando preguntas que ayudasen a la reflexión de los futuros profesores de ciencia y que, por tanto, sirvieran de andamios para facilitar la evolución de sus ideas sin imponerlas ${ }^{10}$.

De manera general, se puede decir que los educadores usaron tres estrategias durante la fase intermedia de la actividad: la generación de conflictos cognitivos, la formulación de nuevas preguntas

Tabla 4. Aspectos epistémicos de NDC relativos a las cuatro controversias de HDC implementadas.

\begin{tabular}{|c|c|c|c|c|c|c|}
\hline \multirow[t]{2}{*}{ Controversias } & \multirow[t]{2}{*}{$\begin{array}{l}\mathrm{N}^{\circ} \text { de } \\
\text { grupos }\end{array}$} & \multirow[t]{2}{*}{$\begin{array}{l}N^{0} \text { de indicadores } \\
\text { de las rúbricas }\end{array}$} & \multicolumn{2}{|c|}{$\begin{array}{l}N^{\circ} \text { de indicadores } \\
\text { citados }\end{array}$} & \multicolumn{2}{|c|}{$\begin{array}{l}N^{0} \text { de veces que se } \\
\text { citan los indicadores }\end{array}$} \\
\hline & & & Inicial & Final & Inicial & Final \\
\hline Semmelweis & 4 & 24 & 17 & 20 & 25 & 52 \\
\hline Fermentación & 8 & 28 & 21 & 26 & 58 & 99 \\
\hline $\mathrm{ADN}$ & 4 & 16 & 10 & 12 & 19 & 38 \\
\hline Generación espontánea & 4 & 22 & 13 & 19 & 23 & 56 \\
\hline TOTAL & 20 & 90 & $61(68 \%)$ & $77(86 \%)$ & 125 & 245 \\
\hline
\end{tabular}

Fuente: elaboración propia de los autores.

10. Cabe recordar, al respecto, que las posturas dogmáticas en educación solo logran que los estudiantes declaren lo que se espera de ellos, en el mejor de los casos, sin que realmente integren las nuevas ideas en sus esquemas mentales. 
Tabla 5. Fragmentos de los argumentos usados en las respuestas de los informes finales de los grupos a tres cuestiones epistémicas de la controversia entre Pasteur y Pouchet sobre la generación espontánea.

\begin{tabular}{|c|c|}
\hline $\begin{array}{l}\text { Contenidos epistémicos de NDC } \\
\text { de las cuestiones }\end{array}$ & $\begin{array}{l}\text { Fragmentos de algunas respuestas finales de los grupos con alusiones argumentadas a } \\
\text { los descriptores }\end{array}$ \\
\hline $\begin{array}{l}\text { C1. Papel de las creencias } \\
\text { teóricas en la interpretación de } \\
\text { las observaciones. }\end{array}$ & $\begin{array}{l}\text { "[...] la aparición o no de organismos vivos tras un experimento, cada uno [Pasteur y } \\
\text { Pouchet] lo interpretaba de una forma distinta, apareciese vida o no era explicable, de } \\
\text { manera que siempre su teoría era la válida". } \\
\text { "[...] Pasteur rechazaba de plano la teoría de la generación espontánea, si un experi- } \\
\text { mento fallaba y en el matraz usado aparecían microorganismos, argumentaba que había } \\
\text { una contaminación accidental, sin evidencias directas de esto, y lo mismo podría decirse } \\
\text { de Pouchet". }\end{array}$ \\
\hline $\begin{array}{l}\text { C2. Papel de los diseños } \\
\text { experimentales en los resultados } \\
\text { obtenidos. }\end{array}$ & $\begin{array}{l}\text { "Tuvo mucha importancia el uso de la infusión de heno (como hacía Pouchet), en lugar } \\
\text { de utilizar infusión de levadura (como hizo Pasteur), ya [que] la temperatura idónea para } \\
\text { la total eliminación de los microorganismos en la muestra de heno es de } 120^{\circ} \mathrm{C} \text { y de } 100 \\
{ }^{\circ} \mathrm{C} \text { en la de levadura, lo que no se conocía en la época". } \\
\text { "[...] las variables estudiadas tenían influencia en los resultados y el control de esas va- } \\
\text { riables no se tuvo en cuenta, no solo en la elección de la muestra, sino también en los } \\
\text { lugares en los que se hacía la experiencia cuando se quiso estudiar la influencia del aire". } \\
\text { "[...] no existía forma de demostrar [directamente] si había o no microorganismos en el } \\
\text { aire, por lo que necesitaban usar métodos indirectos, lo que hace la interpretación de los } \\
\text { resultados más compleja". } \\
\text { "Pasteur quería demostrar que la generación espontánea no se producía, era falsa, y esto } \\
\text { no es lo mismo que demostrar, como pretendía Pouchet, que una teoría es cierta". }\end{array}$ \\
\hline C3. Experimentos cruciales. & $\begin{array}{l}\text { "En } 1876 \text { Bastian demostró que en el extracto hervido de heno se encontraban espo- } \\
\text { ras termoestables de bacilos que empezaban a desarrollarse con el suministro de oxí- } \\
\text { geno y que por lo tanto son resistentes al calor, datos que en el momento del debate se } \\
\text { desconocían". } \\
\text { "[...] el mismo Pasteur tuvo que aceptar el sesgo experimental de los diseños en su disputa } \\
\text { con Pouchet; teniendo que rediseñar los experimentos para asegurar que se alcanzaban } \\
\text { los } 120^{\circ} \mathrm{C}^{\prime} . \\
\text { "[...] Tyndall demostró el papel del aire como vector de microorganismos ya que consi- } \\
\text { guió esterilizarlo, verificar que lo estaba, y de esta manera impedir que los líquidos orgá- } \\
\text { nicos esterilizados se alterasen". } \\
\text { "[...] esto deja una pregunta sin contestar, y es la procedencia del primer ser vivo, cues- } \\
\text { tión de fondo siempre presenta en la teoría del a generación espontánea, y motivo por el } \\
\text { que quizás la creencia fue tan persistente". }\end{array}$ \\
\hline
\end{tabular}

Fuente: elaboración propia de los autores.

para enriquecer los debates y la introducción de información adicional en forma de ejemplos de otros casos de HDC. La efectividad de estas estrategias puede ser valorada a partir de los resultados finales mostrados en la tabla 4.

Un ejemplo de ello fue la discusión del significado y papel del error en el contexto de la controversia entre Pasteur y Liebig. Lo siguiente es un extracto de lo que se discutió en clase al respecto, y cómo el educador trató de encauzar el debate hacia ideas mejor informadas por medio de preguntas auxiliares para generar conflictos cognitivos principalmente ${ }^{11}$ :

EM12: [...] La única forma que hay de profundizar experimentalmente es llegando a equivocarte. Si no te equivocas y aciertas a la primera, pues estupendo. Pero normalmente es ensayo y error [...].

11. ED es educador. EM estudiante del MAES. 
ED: ¿Estás intentando decir que cuando se hace un experimento, si el investigador acierta a la primera con su hipótesis, se debe dar por satisfecho?

EM12: Bueno, es que es muy difícil que se compruebe a la primera.

ED: Y el error, ¿cómo lo detecta el investigador? ¿Sabe cuándo ha tenido un error?

EM11: En algunas ocasiones lo sabe, y si no, seguramente se lo dirán otros.

ED: ¿Puedes poner un ejemplo?

EM11: Por ejemplo, cuando estás en el laboratorio haciendo una reacción química y quieres obtener un resultado final, pero luego no lo obtienes.

ED: Eso es porque tú tienes un marco teórico en la cabeza y sabes lo que debería obtenerse, ¿no? Pero, ¿y si no existe un marco teórico sólido? En el caso de la controversia no había un marco teórico previo consistente, de hecho, Liebig y Pasteur estaban siendo los precursores. Además, ninguno de los dos llegó a explicar bien el fenómeno de la fermentación.

EM4: Yo no entiendo a qué nos referimos con error en este contexto porque ellos [Pasteur y Liebig] no se estaban equivocando. Ellos estaban malinterpretando en función de lo que pensaban filosóficamente. En la época de estos señores había una discusión en todos los ámbitos de la ciencia entre el mecanicismo y el vitalismo. Así que ellos no se equivocaron, sino que dijeron: "sobre lo que yo sé, esto es lo que puedo concluir".

ED: ¿Creéis que ellos pensaron que estaban equivocados en algún momento? Porque nosotros tenemos la ventaja de la perspectiva del tiempo para juzgar sus propuestas [...]

EM15: Tendemos a pensar que el error es algo malo, y en la ciencia realmente un error es un avance, te hace reconducir tus investigaciones [...]

EM5: La prueba de que las interpretaciones de Pasteur y Liebig no eran un error total es que sirvieron de punto de partida para las investigaciones posteriores sobre el fenómeno [de la fermentación].

EM4: Yo es que pienso que los dos tenían razón.

EM8: A ver, la parte explicativa de sus teorías no era correctas. Habían hecho experimentos interesantes, pero no fueron bien explicados [...]
EM4: Pienso que cada uno dio una explicación parcial del fenómeno... Entonces, si juntas las dos... cuando se muele el fermento o la levadura encuentras que lo que hay dentro es una reacción química, que era lo que proponía Liebig. Pero al final necesita la participación de un ser vivo; con lo cual, es una superposición de explicaciones evidentemente mejorable. Porque eso es lo bueno de la ciencia, que como se construye por capas, al final llega uno [se refiere a Büchner] que te limpia de filosofía toda la explicación anterior y da una explicación científica más correcta que las de Pasteur y Liebig.

\section{Conclusiones}

Las conclusiones que se pueden extraer del análisis de los cuatro estudios realizados son las siguientes:

1) La lectura inicial de la narración en grupos pequeños permitió que los futuros profesores de ciencia hicieran alusión, en los argumentos de sus informes, a más de dos tercios de los indicadores epistémicos de las rúbricas. El número de veces que se citaron estos indicadores fue, globalmente, alrededor de dos. Si bien variaron entre las diversas controversias, siendo mayor en el caso de la fermentación, en valor absoluto, que en las otras tres.

2) En los argumentos de sus informes finales los futuros profesores de ciencia hicieron alusión a más de cuatro de cada cinco indicadores epistémicos de las rúbricas. El número de veces que se citaron estos indicadores fue, globalmente, alrededor de 3; aunque variaron entre las diversas controversias, siendo otra vez mayor en la de la fermentación, en valor absoluto, que en las otras tres. Estos resultados son indicativos de la incidencia positiva que tuvo la segunda fase en gran grupo. A pesar de que los resultados ya fueron bastante aceptables en la fase inicial, al concluir la actividad los futuros profesores de ciencia fueron capaces de aportar argumentos más variados, y en mayor cantidad, para razonar sus ideas sobre las cuestiones planteadas. Ello evidenció un progreso considerable en su capacidad de argumentación al respecto. 
En resumen, podemos destacar el valor didáctico de la metodología cualitativa empleada para enseñar sobre los aspectos epistémicos de NDC seleccionados. En el contexto de las distintas controversias de HCD empleadas, se promovió que los estudiantes analizaran críticamente su contenido $y$, en respuestas a preguntas abiertas y reflexivas, argumentaran sus puntos de vista sobre los aspectos de NDC discutidos en el contexto de las mismas. Todo ello en un clima de discusión y debate entre los estudiantes (tanto en pequeño grupo como en gran grupo), con la orientación apropiada y esencial del educador, para favorecer la progresión o enriquecimiento de tales argumentos, conforme a las ideas epistémicas sobre NDC mejor informadas ${ }^{12}$.

Por último, consideramos que son necesarios más estudios empíricos en el aula para seguir profundizando en los aspectos epistémicos tratados, o en otros que pudieran considerarse necesarios incluir.

\section{Referencias}

Acevedo-Díaz, J. A. (2008). El estado actual de la naturaleza de la ciencia en la didáctica de las ciencias. Revista Eureka sobre Enseñanza y Divulgación de las Ciencias, 5(2), 134-169. DOI: https://doi.org/10.25267/Rev Eureka ensen divulg cienc.2008.v5.i2.02

Acevedo-Díaz, J. A. (2009). Enfoques explícitos versus implícitos en la enseñanza de la naturaleza de la ciencia. Revista Eureka sobre Enseñanza y Divulgación de las Ciencias, 6(3), 355-386. DOI: https://doi.org/10.25267/Rev Eureka ensen divulg cienc.2009.v6.i3.04

Acevedo-Díaz, J. A. (2017). Sobre la naturaleza de la ciencia y los contextos científicos de descubrimiento y justificación. En Divulgación y Cultura Científica Iberoamericana. Recuperado de http://www.oei.es/historico/divulgacioncientifica/?Sobre-la-naturaleza-de-la-ciencia-y-los-contextos-cientificos-de-descubrimiento

Acevedo-Díaz, J. A. (2018). ¿Naturaleza de la ciencia o naturaleza de las ciencias? En Divulgación y Cultura Científica Iberoamericana. Recuperado de http://www.oei.es/historico/ divulgacioncientifica/?Naturaleza-de-la-ciencia-o-naturaleza-de-las-ciencias

Acevedo-Díaz, J. A. y García-Carmona, A. (2016a). Algo antiguo, algo nuevo, algo prestado. Tendencias sobre la naturaleza de la ciencia en la educación científica. Revista Eureka sobre Enseñanza y Divulgación de las Ciencias, 13(1), 3-19. DOI: http://hdl.handle.net/10498/18010

Acevedo-Díaz, J. A. y García-Carmona, A. (2016b). Rosalind Franklin y la estructura del ADN: un caso de historia de la ciencia para aprender sobre la naturaleza de la ciencia. Revista Científica, 27, 162-175. DOI: https://doi. org/10.14483/udistrital.jour.RC.2016.25.a2

Acevedo-Díaz, J. A. y García-Carmona, A. (2016c). Uso de la historia de la ciencia para comprender aspectos de la naturaleza de la ciencia. Fundamentación de una propuesta basada en la controversia Pasteur versus Liebig sobre la fermentación. Revista Iberoamericana de Ciencia, Tecnología y Sociedad, 11(33), 203-226.

Acevedo-Díaz, J. A. y García-Carmona, A. (2017). Controversias en la historia de la ciencia y cultura científica. Madrid: Los Libros de la Catarata.

Acevedo-Díaz, J. A., García-Carmona, A. y Aragón-Méndez, M. M. (2016a). La controversia Pasteur vs. Pouchet sobre la generación espontánea: un recurso para la formación inicial del profesorado en la naturaleza de la ciencia desde un enfoque reflexivo. Ciência \& Educação, 22(4), 913-933. DOI: https://doi. org/10.1590/1516-731320160040006

12. Las personas interesadas en conocer las mejoras relativas a la comprensión de los aspectos epistémicos por niveles, así como de los indicadores de las rúbricas más y menos citados en los argumentos de los informes iniciales y finales, pueden consultar los capítulos $7,8,9$ y 11 de Acevedo-Díaz, García-Carmona y Aragón-Méndez (2017a). 
Acevedo-Díaz, J. A., García-Carmona, A. y Aragón-Méndez, M. M. (2016b). Un caso de Historia de la Ciencia para aprender Naturaleza de la Ciencia: Semmelweis y la fiebre puerperal. Revista Eureka sobre Enseñanza y Divulgación de las Ciencias, 13(2), 408-422. DOI: http://hdl.handle.net/10498/18297

Acevedo-Díaz, J. A., García-Carmona, A. y Aragón-Méndez, M. M. (2017a). Enseñar y aprender sobre naturaleza de la ciencia mediante el análisis de controversias de historia de la ciencia. Resultados y conclusiones de un proyecto de investigación didáctica. Madrid: OEI.

Acevedo-Díaz, J. A., García-Carmona, A. y Aragón-Méndez, M. M. (2017b). Historia de la ciencia para enseñar naturaleza de la ciencia: una estrategia para la formación inicial del profesorado de ciencia. Educación Química, 28(3), 140-146.

Allchin, D. (2011). Evaluating Knowledge of the Nature of (Whole) Science. Science Education, 95(3), 518-542. DOI: https://doi.org/10.1002/ sce.20432

Clough M.P. (2011). The Story Behind the Science: Bringing Science and Scientists to Life in Post-Secondary Science Education. Science \& Education, 20(7-8), 701-717. DOI: https://doi. org/10.1007/s11191-010-9310-7

Dagher, Z. R. y Erduran, S. (2016). Reconceptualizing the nature of science for science education. Why does it matter? Science \& Education, 25(1-2), 147-164. DOI: https://doi. org/10.1007/s11191-015-9800-8

Driver, R., Leach, J., Millar, R. y Scott, P. (1996). Young People's Images of Science. Buckingham, Reino Unido: Open University Press.

Hodson, D. (2014). Nature of Science in the Science Curriculum: Origin, Development, Implications and Shifting Emphases. En M. R. Matthews (ed.), International Handbook of Research in History, Philosophy and Science Teaching (pp. 911-970). Dordrecht: Springer. DOI: https://doi. org/10.1007/978-94-007-7654-8 28
Hodson, D. y Wong, S. L. (2017). Going Beyond the Consensus View: Broadening and Enriching the Scope of NOS-Oriented Curricula. Canadian Journal of Science, Mathematics and Technology Education, 17(1), 3-17. DOI: https://doi.org/10.1080/14926156.2016.1271 $\underline{919}$

Horizonte 2020 para la Investigación e Innovación en la Unión Europea (s.f.). Ciencia con y para la Sociedad. Recuperado de https://eshorizonte2020.es/mas-europa/ ciencia-con-y-para-la-sociedad

Laherto, A., Kampschulte, L., de Vocht, M., Blonder, R. Akaygun, S. y Apotheke, J. (2018). Contextualizing the EU's "Responsible Research and Innovation" Policy in Science Education: A Conceptual Comparison with the Nature of Science Concept and Practical Examples. Eurasia Journal of Mathematics, Science and Technology Education, 14(6), 2287-2300. DOI: https://doi.org/10.29333/ ejmste/89513

Lederman, N. G. (2007). Nature of science: past, present, and future. En S. K. Abell y N. G. Lederman (eds.), Handbook of Research on Science Education (pp. 831-879). Mahwah, EE. UU.: Lawrence Erlbaum Associates.

Martins, A. F. P. (2015). Natureza da Ciência no ensino de ciências: uma proposta baseada em "temas" e "questões". Caderno Brasileiro de Ensino de Física, 32(3), 703-737. DOI: https://doi.org/10.5007/2175-7941.2015v32n $\underline{3 p 703}$

Next Generation Science Standards (NGSS) (2013). The Next Generation Science Standards: For States, by States. Washington D.C.: National Academy of Sciences Press.

Norris, S. P. y Ennis, R. H. (1989). Evaluating Critical Thinking. The Practitioners' Guide to Teaching Thinking Series. Pacific Grove, EE. UU.: Critical Thinking Press.

Organisation for Economic Co-operation and Development (OCDE) (2017). PISA 2015 Assessment and Analytical Framework: Science, 
Reading, Mathematic, Financial Literacy and Collaborative Problem Solving, revised edition. París: OCDE.

Seale, C. (1999). The quality of qualitative research. Introducing qualitative methods series. Londres: Sage. DOI: https://doi. org/10.4135/9780857020093
Shamos, M. H. (1995). The myth of scientific literacy. New Brunswick, EE. UU.: Rutgers University Press. Vázquez-Alonso, A. y Manassero-Mas, M. A. (2018). El conocimiento epistémico en la evaluación de la competencia científica en PISA 2015. Revista de Educación, 380, 103-128. DOI: $\underline{10.4438 / 1988-592 X-R E-2017-380-373}$ 